\title{
Treatment of Painful Limbs/Moving Extremities with Botulinum Toxin Type A Injections
}

\author{
Mahmood Eisa ${ }^{a, c} \quad$ Carlos Singer $^{b}$ Cenk Sengun ${ }^{b} \quad$ Angela Russel $^{b} \quad$ Bahman Jabbari $^{a}$ \\ Spiridon Papapetropoulos ${ }^{b}$ \\ ${ }^{a}$ Department of Neurology, Yale University School of Medicine, New Haven, Conn., ${ }^{b}$ Department of Neurology, \\ University of Miami Leonard M. Miller School of Medicine, Miami, Fla., and ' Neurology, Neurosurgery and \\ Spine Clinic of South Georgia, Valdosta, Ga., USA
}

Dear Sir,

The syndrome of painful legs/moving toes (PLMT) described first by Spillane et al. [1] in 1971 depicts a clinical condition characterized by involuntary foot and toe movements associated with pain in the affected feet and lower legs. In the typical variant of PLMT pain is usually the first symptom, preceding the onset of movement disorder by days to years, although the reverse sequence may occur. Other clinical variants may be limited to one foot [2], affect the upper limbs - painful arms/ moving fingers [3], or lack pain (painless legs/moving toes) [4]. Since the periodic involuntary movements may affect both upper and a lower limbs the term painful limbs/moving extremities (PLME) has been suggested for this movement disorder. Although the etiology of PLME remains unclear, several precipitating factors have been identified: many patients demonstrate clinical and electrophysiological evidence of peripheral nervous system injury with electromyographic changes with periodic myokymic discharges in the affected muscles $[5,6]$. It is currently believed that the central nervous system may play an important role in PLMT through the presence of a central oscillator [5].

In some patients with PLME the pain can be severe and unbearable, failing high doses of opioids. Treatment is often unsatisfactory with few reports of success with sympathetic block, lumbar epidural blocks or epidural spinal cord stimulation [7-9].

We describe herein 2 patients with PLMT in whom analgesic multipharmacy failed to relieve their severe lower limb pain. On the other hand treatment with Botulinum toxin type A (Botox ${ }^{\circledR}$, Allergan, Inc.) resulted in both pain relief and improvement of involuntary movements.

\section{Case 1}

A 62-year-old male with a history of L5-S1 vertebral fusion for chronic back pain was evaluated for lower back 'spasms', cramps and severe pain in the lower legs and involuntary toe movements. He reported a 1-year history of pain in the foot and lower legs with 'very uncomfortable feeling inside the leg muscles, constant movement and burning/electrical sensations in the lower legs'. The pain and foot movements were most severe in supine position, interfering with his rest and sleep. His treatment included gabapentin, baclofen, trazodone, oxycodone, alprazolam, mirtazapine and paracetamol.

On examination, continuous semirhythmic, bilateral movements of the toes, feet and lower leg were noted. The toes and feet moved in all directions but the most sustained pattern of movement was flexion/extension. The movement showed no diurnal pattern, worsened with stress and disappeared during sleep. The patient's neurological examination was otherwise normal.

Botulinum toxin type A (Botox), 100 units $/ \mathrm{ml}$, was injected into those toe and lower leg muscles which on inspection and palpation showed the most sustained pattern of contraction (foot plantar flexion and toe flexion): 45 units into each flexor digitorum brevis, 50 units into each gastrocnemius divided into 25 units medial head and 25 units lateral head and 60 units into paraspinal muscles on each side; 30 units at L5, and 30 units at S1 level (for chronic back pain management). Palpation and observation of active muscles is a well-accepted method for botulinum toxin injections [6]. The patient's baseline medications were not changed during botulinum toxin treatment. We performed clinical evaluation and obtained videotapes before injection and at 3 days, and 3 months after injection (online suppl. video 1, www.karger.com/doi/10.1159/000 138962).

The 4-point Clinical Global Impression scale (a classic instrument for making

\section{KARGER}

Fax +4161306 1234 E-Mail karger@karger.ch www.karger.com
Spiridon Papapetropoulos, MD, PhD, Associate Prof. of Neurology Division of Movement Disorders, Department of Neurology, University of Miami Miller School of Medicine, 1501 NW 9th Avenue (NPF), Room 2017 Miami, FL 33136 (USA)

Tel. +1 305243 8461, Fax +1 305243 3321, E-Mail spapapetropoulos@med.miami.edu 
global assessments) as completed by the treating physician was used to measure the patient's response to botulinum toxin $\mathrm{A}$ injections $(0=$ no benefit, $1=$ mild, $2=$ moderate, 3 = marked). The Clinical Global Impression scale is frequently used in medical care and clinical research because of its face validity and practicability [10]. The injections resulted in marked relief of leg pain and marked reduction of foot and toe movements in 3 days. At 2 weeks the movements were barely visible. At 3 months moderate amount of toe movements were noted (still less than pretreatment) with some pain returning (50-60\% of preinjection). No muscle weakness was reported or noted.

\section{Case 2}

A 76-year-old female was evaluated for involuntary toe movements and painful feet. She had a 7-year history of bilateral, asymmetric, involuntary flexion of her toes (right $>$ left) of gradual onset. Three years ago, she began to experience pain in the distal part of her feet (plantar and dorsal surface) accompanied by a sensation of numbness in the same areas. She was originally diagnosed and treated for atypical restless legs syndrome with dopamine agonists and subsequently gabapentin without success. Her past medical history was significant for a diagnosis of systemic lupus erythematosus, atrial fibrillation and hyperthyroidism. Her medications included diltiazem $\mathrm{HCl}$, metoprolol tartrate, Coumadin, temazepam, methimazole, propylthiouracil and a combination of chlordiazepoxide and amitriptyline. Neurological examination was normal except for reduced sensory perception to pinprick in the distal third of the right foot in the dorsal aspect, and reduced perception of vibration in the toes of the left foot. Inspection of the feet disclosed $(2-3 \mathrm{~Hz})$ irregular, bilateral, asymmetric (right $>$ left) predominantly flexion movements of the toes with little foot movement. Movements were exacerbated with stress and disappeared during sleep.

Electrophysiological studies (NCV, EMG) included studies on the bilateral tibial motor, bilateral median (motor and sensory), bilateral ulnar (motor and sensory) (and corresponding F waves) and bilateral sural sensory nerves. A selected group of muscles were sampled with disposable concentric needle electrode to evaluate spontaneous activity, insertional activity, motor unit configuration and recruitment pattern in lower extremity muscles. The paraspinal muscles were not evaluated as the patient was on warfarin. There was electroneurophysiological evidence for: (a) peripheral sensory motor axonal neuropathy resulting in significant sensory axon loss affecting legs more than arms; (b) right median nerve dysfunction at the wrist (carpal tunnel) is resulting in significant axon loss; (c) right ulnar nerve dysfunction at the elbow contributing to underlying significant sensory axonal loss. A lumbosacral spine MRI revealed intervertebral disk disease at L4-L5 with moderately severe canal and neuroforaminal stenosis.

The patient initially received low doses of botulinum toxin type A (Botox, 12.5 units/muscle) in each flexor digitorum longus with improvement $(30 \%)$ of both intensity of pain and toe movements. During the following session (3 months later) she was injected with 15 units in the right and 12.5 units in the left flexor digitorum longus. During her third session the patient received 25 units in each muscle. According to the Clinical Global Impression scale completed by the treating physician a mild improvement in pain intensity and a moderate improvement in involuntary movements was noted. The patient's baseline medications were not changed during botulinum toxin treatment. No muscle weakness or other side effects were reported.

\section{Discussion}

Several modes of treatment have been used for PLME with variable results. Oral medications such as baclofen, benzodiazepines, tricyclic antidepressants, anticonvulsant, beta-blockers, and corticosteroids usually provide no significant benefit in PLME [7]. Progabide, a $\gamma$-aminobutyric acid receptor agonist [8], and gabapentin [7] have been used with some success. Among interventional procedures, lumbar sympathetic block provided transient relief in about $50 \%$ of patients [5], and lumbar epidural block [9] or epidural spinal cord stimulation [11] provided some measure of benefit to PLME patients. Benefit from botulinum toxin type A injections has been reported previously in a single case of the painless arms/moving fingers variant of PLME [12].
Botulinum toxin type A has been widely used in hyperkinetic movement disorders and especially dystonia with remarkable success. There is also extensive evidence suggesting that it may provide relief in human pain disorders (migraine, myofacial pain syndrome) [13]. Furthermore, results from studies in rodents suggest that it may be useful in neuropathic pain [14].

We selected muscles for injection based on observation of the movement pattern and palpation of contractions. The site of pathology in our first patient was most likely at the level of lumbosacral roots and for our second patient at the level of peripheral nerves. Previous reviews of literature on PLMT indicated these sites in a number of PLME patients $[1,5,7,9]$. The cause of PLMT of our patients remains unclear although the association with lumbosacral root injury (patient 1) and peripheral neuropathy (patient 2) should be considered. A peripheral neuropathy workup, however, failed to determine an exact cause for the latter but contributions from lupus and/or thyroid disease remain viable possibilities. In patient 1 , the foot movements had a complex pattern including medial and lateral movements, yet injection into toe and foot flexors improved movements. We think the most likely explanation for this is activation by proximity, for example secondary activation of tibialis posterior muscle (foot inverter, which is anatomically placed next to gastrocnemius).

Our data shows that in some patients with PLMT, administration of botulinum toxin can cause significant pain relief and reduction of involuntary foot and toe movements. The mechanism is not well understood, however, animal studies suggest a number of mechanisms: reduction of muscle spindle discharge leading to decreased activity of gamma loop and central sensitization; antisympathetic, antiglutamergic and anti-inflammatory effects and inhibition of local pain neurotransmitters [15]. The role of botulinum toxin injections in this disorder warrants further investigation in larger cohorts. 
-1 Spillane JD, Nathan PW, Kelly RE, Marsden CD: Painful legs and moving toes. Brain 1971;94:541-556.

-2 Sanders P, Waddy HM, Thompson PD: An 'annoying' foot: unilateral painful legs and moving toes syndrome. Pain 1999;82:103104.

3 Supiot F, Gazagnes MD, Blecic SA, Zegers de Beyl D: Painful arm and moving fingers: clinical features of four new cases. Mov Disord 2002;17:616-618.

$\checkmark 4$ Walters AS, Hening WA, Shah SK, Chokroverty S: Painless legs and moving toes: a syndrome related to painful legs and moving toes? Mov Disord 1993;8:377-379.

5 Jabbari B, Molloy FM, Erickson M, Floeter MK: Bilateral painful hand-moving fingers: electrophysiological assessment of the central nervous system oscillator. Mov Disord 2000;15:1259-1263.
6 Jankovic J: Needle EMG guidance for injection of botulinum toxin: needle EMG guidance is rarely required. Muscle Nerve 2001; 24:1568-1570.

7 Villarejo A, Porta-Etessam J, Camacho A, Gonzalez De La Aleja J, Martinez-Salio A, Penas M: Gabapentin for painful legs and moving toes syndrome. Eur Neurol 2004;51: 180-181.

$>8$ Bovier P, Hilleret H, Tissot R: Progabide treatment of a case of the syndrome of painful legs and moving toes (in French). Rev Neurol (Paris) 1985;141:422-424.

$\checkmark 9$ Okuda Y, Suzuki K, Kitajima T, Masuda R, Asai T: Lumbar epidural block for 'painful legs and moving toes' syndrome: a report of three cases. Pain 1998;78:145-147.
10 Guy W: Clinical Global Impression; in ECDEU Assessment Manual for Psychopharmacology, revised. Rockville, National Institute of Mental Health, 1976.

11 Takahashi H, Saitoh C, Iwata O, Nanbu T, Takada S, Morita S: Epidural spinal cord stimulation for the treatment of painful legs and moving toes syndrome. Pain 2002;96: 343-345.

12 Singer C, Papapetropoulos S: A case of painless arms/moving fingers responsive to botulinum toxin a injections. Parkinsonism Relat Disord 2007;13:55-56.

13 Bhidayasiri R, Truong DD: Expanding use of botulinum toxin. J Neurol Sci 2005;235:1-9.

14 Bach-Rojecky L, Relja M, Lackovic Z: Botulinum toxin type a in experimental neuropathic pain. J Neural Transm 2005;112:215219.

15 Lang AM: Considerations for the use of botulinum toxin in pain management. Lippincotts Case Manag 2006;11:279-282. 